\title{
Friending adolescents on social networking websites: a feasible research tool
}

\author{
Libby N Brockman*, Dimitri A Christakis and Megan A Moreno
}

\begin{abstract}
Objective: Social networking sites (SNSs) are increasingly used for research. This paper reports on two studies examining the feasibility of friending adolescents on SNSs for research purposes.

Methods: Study 1 took place on www.MySpace.com where public profiles belonging to 18-year-old adolescents received a friend request from an unknown physician. Study 2 took place on www.Facebook.com where college freshmen from two US universities, enrolled in an ongoing research study, received a friend request from a known researcher's profile. Acceptance and retention rates of friend requests were calculated for both studies.

Results: Study 1: 127 participants received a friend request; participants were 18 years-old, $62.2 \%$ male and 51.8\% Caucasian. 49.6\% accepted the friend request. After 9 months, 76\% maintained the online friendship, 12.7\% defriended the study profile and $11 \%$ deactivated their profile. Study 2: 338 participants received a friend request; participants were 18 years-old, 56.5\% female and $75.1 \%$ Caucasian. 99.7\% accepted the friend request. Over 12 months, 3.3\% defriended the study profile and $4.1 \%$ deactivated their profile. These actions were often temporary; the overall 12-month friendship retention rate was $96.1 \%$.
\end{abstract}

Conclusion: Friending adolescents on SNSs is feasible and friending adolescents from a familiar profile may be more effective for maintaining online friendship with research participants over time.

Keywords: Adolescent; Social networking website; Online friend; Friending; Internet; Social media; Research methods

\section{Background}

Social networking websites (SNSs) are virtual communities in which users create individual web profiles, display personal contact information and post a variety of behaviors and emotions in real time; they are tremendously popular among adolescents and young adults [1-3]. To date, SNSs have been used as a research tool to recruit, identify, or target participants [4-6]. There are two major advantages of using SNSs for research purposes. First, SNSs are nearly ubiquitous among adolescents and therefore present an opportunity for researchers to consistently and frequently interact with the majority of adolescents worldwide. Almost three-quarters (73\%) of adolescents between the ages of 12 and 17 years and up to $97 \%$ of college students use SNSs. Further, the majority of these users report daily use of such websites $[3,7,8]$.

\footnotetext{
* Correspondence: libby.brockman@seattlechildrens.org

Seattle Children's Research Institute, Center for Child Health Behavior and Development, 2001 8th Avenue, Suite 400, M/S CW8-6, PO Box 5371, Seattle, WA 98121, USA
}

Second, many adolescent profile owners display personal demographic information on their SNS profiles, including gender, age, race or ethnicity, relationship status, sexual orientation, level of education, hometown, and current geographical location [9-11]. This information can be used for identification and recruitment of research participants. SNSs may also offer the ability to selectively target adolescents who display risk behaviors on their profiles. Previous research has shown that more than $50 \%$ of 18 -year-old's MySpace profiles display references to risk behaviors that are concerning to clinicians, parents and educators, including alcohol use (37\%), sexual activity (24\%), violence (14.4\%), tobacco use (13\%), and substance use (10\%) [12]. Displays of these risk behaviors have been linked to offline engagement in these behaviors $[13,14]$. Thus, SNSs may be useful for identifying and targeting interventions towards high risk populations of adolescents who engage in risky behaviors offline.

\section{Springer}

(c) 2014 Brockman et al.; licensee Springer. This is an Open Access article distributed under the terms of the Creative Commons Attribution License (http://creativecommons.org/licenses/by/2.0), which permits unrestricted use, distribution, and reproduction in any medium, provided the original work is properly credited. 


\section{Current challenge}

The use of public SNS profiles is consistent across previous studies investigating SNSs. SNSs allow users to control the extent to which their profile content is viewable to the general public. Profile security settings can be set as public, meaning that the majority of profile content is available to the general SNS population, or private, meaning that full profile content is only available to users who have designated each other as a SNS friend through the friending process. Some SNS users allow their profile to be publicly viewable to others in their school, education, or employee networks, or to friends of friends. Further, many SNSs allow profile owners to customize their profile security settings on an individual basis within their existing online friend group.

As internet safety and online privacy concerns arise, current trends suggest many SNS users are moving away from public profile settings towards private, customized profile settings $[15,16]$. Private profiles are not accessible to the general SNS user and with fewer publicly available SNS profiles, using SNSs for research purposes may be less feasible because demographic and contact information, as well as behavior and intention information, is not viewable. With fewer public profiles, researchers no longer have access to adolescent profiles and the informative content therein. This may limit the use of SNSs as a useful research tool.

While some SNS users still maintain public profile settings, it remains unknown whether individuals who select public profile settings differ from those that select private security settings, and whether the content on public and private profiles varies. Therefore, while research has been conducted using publicly available profiles, those results may not be generalizable to individuals who maintain a private SNS profile.

In order to optimize SNSs' full potential as a research tool, innovative approaches to using SNSs are necessary. As SNS users trend towards increased online privacy, friending research participants on SNSs may provide researchers with a novel and useful research method. Friending involves sending friend requests (FRs) from one profile to another. Upon receiving a FR, a SNS user has the ability to view the profile of the requestor and can then accept, ignore or deny the FR. Accepting a FR is completely voluntary. Once a FR is accepted, the two profiles become linked and both users have mutual access to each other's profiles and the contents found therein. Profiles can become un-linked by "unfriending" the linked profile at any time. Thus, by using the friending function to link profiles with friends, peers, colleagues, acquaintances and strangers, SNS users create expansive online networks and communities.

As far as the authors are aware, there are no documented reports on the methodology of friending participants on
SNSs for research purposes, nor literature that provides average recruitment and retention rates as benchmarks for researchers to use. Therefore, we present two studies investigating SNS friending as a novel research tool, illustrating that this is a feasible tool for different sites and at different time points. The first study investigated adolescents' willingness to accept a FR from an unknown health professional on MySpace, and the second study examined the feasibility of friending adolescents on Facebook (FB) from a known research profile. Overall, this paper aims to report average recruitment and retention rates as benchmarks for future research efforts. We hypothesize that the creation of research SNS profiles and the active friending of research participants is feasible and useful for conducting research on SNSs.

\section{Study 1}

Methods

The primary goal of Study 1 was to assess adolescents' willingness to accept a FR from an unknown physician on the SNS MySpace. The secondary aim of Study 1 was to investigate whether acceptance of a physician's FR was associated with at-risk profile owners, defined as those who displayed sexual references on their profile.

\section{Setting}

Study 1 was conducted between January 2009 and September 2011 and received Institutional Review Board (IRB) approval from the University of Washington. This study was conducted using the SNS MySpace (www.MySpace.com). MySpace was chosen for this study because of evidence that suggests MySpace users may represent a more vulnerable population that is, on average, less educated and of lower socioeconomic status compared to users of Facebook, another popular SNS [3,17-20]. As a goal was to identify future intervention efforts to target more vulnerable adolescent populations that may be less likely to seek care in clinic or receive health messages in school, it was determined that MySpace would be an appropriate venue for this study.

\section{Subject selection}

Study 1 recruited only public MySpace profiles belonging to 18-year-olds living in the United States. Profiles belonging to 18-year-olds were chosen for investigation because this age includes a transition from high school to college and represents a time during which adolescents may engage in risky behaviors [21]. When a MySpace profile is created, users are required to enter a date of birth. The profile owner's age is then calculated in years and displayed among the profile's demographic information. This public display of age was used to identify 18 -year-old profile owners for this study. 
To increase precision, an additional inclusion criterion required profiles to have been accessed by their owner within the past 30 days. This ensured the sample comprised active SNS users such that in the event that a FR was not accepted, it would be due to a participant being unreceptive to the FR rather than an inactive SNS user.

At the time of this study, MySpace featured a browse function that could be used to search for SNS profiles that meet particular criteria. Each individual search provided non-alphabetically and non-numerically listed thumbnail-links to a maximum of 3,000 Web profiles. This browse function was used to search for publicly available MySpace profiles belonging to 18-year-olds. MySpace listed search results in one of several formats: by newest profile, by recently updated profiles, by most recent login date, or by distance from a given zip code. Search results for this study were listed in order of most recent login date.

A total of 191 MySpace profiles were screened in order to identify 127 MySpace users whose profiles fit the inclusion criteria. After identifying 110 profiles that did not display references to sexual behavior ("Non-Displayers"), subsequent Non-Displayers' profiles were considered ineligible $(n=43)$. Other common reasons for profile exclusion included non-public profile settings $(n=8)$ and profiles that stated an age other than 18 years $(n=7)$.

\section{Profile coding}

Each profile was evaluated for the presence ("Displayer") or absence ("Non-Displayer) of displayed references to sexual behavior. All publicly viewable elements of profiles were examined including text, photographs and downloaded icons (i.e., publicly displayed advertisements, cartoons or pictures).

Sexual references were defined using criteria established by the Kaiser Family Foundation and included any depiction of sexual activity or sexually suggestive behavior $[22,23]$. Examples of sexual displays included references to sexual behaviors (i.e. "I'm through with love, thank goodness there's still sex."), photographs depicting partial nudity (i.e. profile owner dressed in lingerie) or intimate personal descriptions (i.e. "Check out what's in my pants").

Profiles were also evaluated for standard demographic variables displayed on all profiles: age, gender and home state. Data was collected on ethnicity, which at the time of this study was typically (approximately $75 \%$ of the time), but not always, available on MySpace profiles given that it was displayed at the profile owner's discretion [12]. Another variable of interest included relationship status; options on MySpace included single, in a relationship, married, engaged, swinger and divorced. Lastly, profile owners' number of online friends was assessed, as these were hypothesized to potentially impact whether the profile owner was willing to accept a FR from a stranger.

Each MySpace profile was viewed one time by the lead investigator. Profiles were not archived, printed nor saved. A $10 \%$ random sub-sample of profiles was coded by an additional investigator within several hours of the initial coding in order to assess inter-rater reliability. This was analyzed using the Cohen's Kappa statistic and the degree of agreement between the two investigators for the variable of sexual references was 0.87 .

\section{Friend request invitation}

For the purpose of this study, a MySpace profile named "Dr Meg" was used. This profile had been created for and used in previous research studies [5]. Content on the Dr Meg profile stated that the profile owner was a 36-year-old female pediatrician interested in adolescent health. In order to verify the validity of the physician, the Dr Meg profile contained accurate information regarding the physician's education, professional credentials, and research interests.

Using this MySpace profile reserved for research purposes, FRs were sent to all Displayers and NonDisplayers in this study. "Acceptors" were profile owners who accepted the FR, and "Non-acceptors" were those who did not. No message was sent to accompany the FR; anecdotal observations suggest it is not uncommon to receive a FR from a stranger without an accompanying message.

All profile owners who accepted the FR for the purpose of this study were later sent a debriefing message via MySpace's internal messaging system. This message was sent after participants accepted the FR; its contents explained why each individual received a FR from the Dr Meg profile and provided more information about the study.

\section{Data analysis}

All statistical analyses were conducted using STATA version 9.0 (Statacorp, College Station, TX). Because relative risk (RR) is a more interpretable summary of association, and because the outcome of accepting a FR is not rare such that odds ratios would not approximate RRs, a modified Poisson regression approach to estimate RRs was used [24,25]. This approach for estimating risk on the basis of binary data does not require that the outcome follow a Poisson distribution. For these purposes, the Poisson model was a "working model" to facilitate the estimation and did not affect the consistency of the RR estimation. Incident rate ratios were calculated, adjusting for gender, ethnicity, and relationship status, to evaluate whether the display of sexual references was associated with willingness to accept a physician's FR. Students t-test were used to compare mean number of MySpace friends between 
Acceptors and Non-Acceptors. Descriptive statistics were calculated, and chi square analyses and Fisher's exact tests were used to calculate differences in proportion of demographic variables between Acceptors and Non-Acceptors as appropriate.

\section{Results}

\section{Sample population}

All 127 profile owners received a FR from the Dr Meg SNS profile. All eligible profile owners were 18 years old and lived in the US, the majority were male (62.2\%) and indicated a relationship status of "single" (60.8\%). Most participants were Caucasian (51.8\%), Hispanic (22.9\%) or Black/African (15.7\%) (Table 1).

\section{FR acceptance rates and retention}

Friend requests were accepted by $49.6 \%$ of profile owners and Acceptors tended to be male (68.3\%), Caucasian (46.5\%), and single (54.2\%). There were no significant demographic differences between Acceptors and Non-Acceptors (Table 1). Among adolescents who accepted the FR and received the debriefing email, all maintained their online friendship with the study profile. After 9 months, 76\% maintained their online friendship with the study profile, $12.7 \%$ defriended the study profile and $11 \%$ deactivated their profile.

\section{Friend acceptance and MySpace displays}

Displayers accounted for $13.4 \%$ of the sample $(n=17)$. The unadjusted estimate of association between FR acceptance and display of sexual references was $R R=3.9$. Adjusting for gender, ethnicity, and relationship status, Acceptors were more than twice as likely as Non-Acceptors to display references to sex on their MySpace profile $(R R=2.25$, 95\% CI: 1.03-4.87, $\mathrm{p}=0.04$ ) (Table 2).

\section{SNS friend counts: acceptors versus non-acceptors}

Overall, profile owners had an average number of 229.5 friends (SD 207.7). There were no significant differences between gender and Displayer vs. Non-Displayer status in regards to a profile owner's number of friends. However, there was a significant difference in mean number of friends between Acceptors (274.0, SD 246.1) and NonAcceptors (186.5, SD 152.3) ( $\mathrm{p}=0.02)$.

\section{Discussion}

Nearly half of the adolescents in Study 1 accepted a FR from an unknown physician's profile, and 76\% maintained that SNS friendship after 9 months. Further, those who accepted the FR were twice as likely to display sexual references on their profile than those who did not accept the FR.

There are several reasons why older adolescents may have chosen to accept the FR. First, the study profile was clearly identified as belonging to a clinician; it contained information to validate the identity of its owner, and included links to the university website for her practice. This may have engendered trust from the adolescents who received the FR. It is important to note that none

Table 1 Study 1: Descriptive characteristics of sample population $(n=127)$

\begin{tabular}{|c|c|c|c|}
\hline & Total $(n=127)$ & Non-Acceptors ${ }^{a}(n=64)$ & Acceptors $^{b}(n=63)$ \\
\hline & \multicolumn{3}{|c|}{ No. profiles (\%) } \\
\hline \multicolumn{4}{|l|}{ Gender } \\
\hline Male & $79(62.2 \%)$ & $36(56.2 \%)$ & $43(68.3 \%)$ \\
\hline Female & 48 (37.8\%) & 28 (43.8\%) & 20 (31.7\%) \\
\hline \multicolumn{4}{|c|}{ Ethnicity $^{c}$ ( $n=83$ displayed ethnicity) } \\
\hline White/Caucasian & $43(51.8 \%)$ & $23(57.5 \%)$ & $20(46.5 \%)$ \\
\hline Latin/Hispanic & $19(22.9 \%)$ & $9(22.5 \%)$ & $10(23.3 \%)$ \\
\hline Black/African & 13 (15.7\%) & $5(12.5 \%)$ & $8(18.6 \%)$ \\
\hline Asian & $2(2.4 \%)$ & 0 & $2(4.7 \%)$ \\
\hline Other & $6(7.2 \%)$ & $3(7.5 \%)$ & $3(6.9 \%)$ \\
\hline \multicolumn{4}{|c|}{ Relationship status $^{c}$ ( $n=120$ displayed relationship status) } \\
\hline Single & $73(60.8 \%)$ & $41(67.2 \%)$ & $32(54.2 \%)$ \\
\hline In a relationship & $34(28.4 \%)$ & $10(16.4 \%)$ & $24(40.7 \%)$ \\
\hline Married & $6(5.0 \%)$ & $5(8.2 \%)$ & $1(1.7 \%)$ \\
\hline Engaged & $5(4.2 \%)$ & $3(4.9 \%)$ & $2(3.4 \%)$ \\
\hline Swinger & $1(0.8 \%)$ & $1(1.65 \%)$ & 0 \\
\hline Divorced & $1(0.8 \%)$ & $1(1.65 \%)$ & 0 \\
\hline
\end{tabular}

${ }^{\mathrm{a}}$ Did not accept Friend Request.

${ }^{\mathrm{b}}$ Accepted Friend Request.

${ }^{\text {cC} C a t e g o r i e s ~ p r o v i d e d ~ b y ~ M y S p a c e ; ~ U s e r s ~ w e r e ~ o n l y ~ a b l e ~ t o ~ s e l e c t ~ a ~ s i n g l e ~ c a t e g o r y . ~}$ 
Table 2 Study 1: Sexual reference display: Acceptors vs Non-Acceptors

\begin{tabular}{|c|c|c|c|c|c|}
\hline & \multicolumn{3}{|c|}{ No. profiles (\% profiles) } & \multirow[b]{2}{*}{ p-value } & \multirow[b]{2}{*}{$\operatorname{RR}(95 \% \mathrm{Cl})^{c}$} \\
\hline & Total $(n=127)$ & Non-Acceptors $^{a}(n=64)$ & Acceptors $^{\mathrm{b}}(n=63)$ & & \\
\hline SNS group: & & & & 0.04 & $2.25(1.03-4.87)$ \\
\hline Non-Displayer ${ }^{d}$ & 110 & $60(55.5 \%)$ & $50(45.5 \%)$ & & \\
\hline Displayer ${ }^{\mathrm{e}}$ & 17 & $4(23.5 \%)$ & $13(76.5 \%)$ & & \\
\hline
\end{tabular}

${ }^{a}$ Non-Acceptors: did not accept Friend Request.

${ }^{b}$ Acceptors: accepted Friend Request.

${ }^{\mathrm{C}}$ Adjusted for gender, ethnicity, relationship status.

${ }^{\mathrm{d} D i d}$ not display references to sexual behavior on MySpace profile.

e Displayed references to sexual behavior on MySpace profile.

of the adolescents in this study had offline connection to or knowledge of the research team or its SNS profile prior to the commencement of this study. Second, adolescents often have several hundred online friends and previous studies suggest these online friendships represent "weak ties" and "loose connections" between individuals [26]. Adolescents may have viewed this FR as an opportunity to establish a higher online friend count and a "weak tie" to the Dr Meg profile. This is supported by the finding that Acceptors had a higher friend count compared to Non-Acceptors; Acceptors may have been more likely to accept a FR from a stranger in order to increase their friend count. Lastly, the FR came from a female profile owner, and most Acceptors were male. Males may have been more likely to accept a FR from a female stranger.

There are two main limitations to this study. First, MySpace users self-report their age on their profile and it was their posted age that was used to select 18 -yearolds for this study. It is possible that participants were either younger or older than the age they posted on their MySpace profile. MySpace users must be at least 13 years old to register for a profile, at least 16 years old to post their age as over 16 and to make their profile public, and users who are under 18 cannot list their age as over 18, nor make changes to their listed age [27]. Thus, it is possible younger adolescents may have registered their profile with an older birth date in order to gain increased access to MySpace. Second, we used online display of sexual references as the only measure of displayed risk behaviors. Previous research shows more than $50 \%$ of 18-year-old's MySpace profiles contain references to other risk behaviors such as alcohol use, substance use and violence [12]. Further research is required to determine whether teens who display these other risk behaviors on their SNS profile are also willing to accept a FR from a physician's SNS profile.

\section{Study 2}

The primary goal of Study 2 was to determine whether friending an adolescent on Facebook was a feasible tool to promote longitudinal connection to participants. The secondary aim was to examine friendship retention rates 12 months later.

\section{Methods}

Setting

Data for Study 2 came from a two-site longitudinal study of substance use among college students. This second study was conducted from May 2011-September 2012 and received IRB approval from Seattle Children's Research Institute and University of Wisconsin-Madison. Study 2 was conducted using the SNS Facebook (www. Facebook.com). Facebook was chosen for this study because at the time, it was the most popular SNS among the college student population [28].

\section{Subjects}

For Study 2, graduated high school students were recruited during the summer before their undergraduate matriculation. Students were randomly selected from the school Registrar's list of incoming freshmen. Inclusion criteria required participants to be incoming freshmen students enrolled at one of two large public universities, 17-19 years of age, and the owner of a Facebook profile. A combination of phone and email were used to recruit participants.

\section{Facebook friend request invitation and consent process}

Two research team profiles were created for this study. Both research profiles were of college student age, self identified as research assistants and were from either of the two universities from which participants were recruited.

During the consent process, participants were informed that they would receive a FR as part of the study's procedures and that this FR would be sent from a research profile. Participants were asked to maintain open security settings with our research team profile throughout the study. All students who consented to participate in the study received a FR.

\section{Data analysis}

All statistical analyses were conducted using STATA version 9.0 (Statacorp, College Station, TX). Descriptive statistics 
were used to calculate the percent of enrolled participants who accepted a FR and the retention of FB friendship status after 12 months.

\section{Results}

\section{Sample population}

A total of 725 incoming freshmen were recruited to participate in this study and 635 were eligible to participate. The majority of ineligible participants was due to not having a working phone number or email address (43.3\%), having switched universities (17.7\%), or not having a Facebook profile (11.1\%). Overall, 338 students consented to participate in the study (54.6\% response rate). Among participants who declined to participate and offered reasons, these reasons included not wanting to start a research study before arriving at college, concern on the part of the parents, and general disinterest in being a research participant. Participants were on average 18 years old, female (56.5\%), and Caucasian (75.1\%). Approximately 60\% of participants were from one university and $40 \%$ from the second university; there were no significant demographic differences between sites (Table 3).

\section{Friend request acceptance rates}

Friend requests were sent to 338 participants and were accepted by $99.7 \%(\mathrm{n}=337)$. Over 12 months, 3.3\% $(\mathrm{n}=11)$ defriended the study profile, of whom $54.5 \%(n=6)$ refriended the study profile at a later point in time. Only $4.1 \%(n=14)$ deactivated their Facebook profile. The overall friendship retention rate after 12 months was $96.1 \%$.

\section{Discussion}

Almost all of the adolescents in Study 2 accepted a FR from a known researcher and almost all of these adolescents

Table 3 Study 2: Descriptive characteristics of sample population $(n=338)$

\begin{tabular}{lc}
\hline Variable & $\mathbf{n}(\%)$ \\
\hline Male & $147(43.5 \%)$ \\
Female & $191(56.5 \%)$ \\
Ethnicity & \\
White/Caucasian & $254(75.1 \%)$ \\
Asian & $40(11.8 \%)$ \\
Multi racial & $19(5.6 \%)$ \\
Latin/Hispanic & $12(3.6 \%)$ \\
Other & $8(2.4 \%)$ \\
Black/African & $5(1.5 \%)$ \\
University & \\
West Coast & $139(41.1 \%)$ \\
Midwest & $199(58.9 \%)$ \\
\hline
\end{tabular}

retained that SNS friendship 12 months later. These results suggest that adolescents are willing to accept a FR on Facebook as part of participation in a research study.

One factor that may have promoted the FR acceptance rates in this study was the fact that prior to sending the FR, the research team alerted participants during the consent process of the impending FR. This means that participants were acquainted with the research team prior to receiving the FR. While it is not uncommon for SNS users to receive FRs from unknown individuals, college students tend to grow their online social networks by primarily adding acquaintances and individuals with whom they share extracurricular activities, and less so than by adding strangers or close contacts [29]. This suggests that college students are more accustomed to being online friends with acquaintances rather than complete strangers. Therefore, the advanced notice of the impending FR may have contributed to high FR acceptance and retention rates because it moved the researcher from "stranger" status to that of "acquaintance".

Limitations of this study include an incomplete understanding of how the study requirement of being Facebook friends may have impacted the response rate of the overall longitudinal study. As only some students described their reasons for not wanting to enroll in a four-year longitudinal study prior to arriving at college, some stated their hesitancy to friend a researcher. However, this was not systematically assessed. Lastly, SNS security options frequently change and SNSs now allow profile owners to customize their profile security settings on an individual basis within one's online friend group. Therefore, even for those participants who accepted the FR, it remains unknown whether they blocked some profile content from our research profile.

\section{General discussion}

Taken together, the results of these two studies suggest that the friending process of SNSs is a feasible research tool and may be particularly helpful for researchers working with adolescents who display risk behaviors online. While the current literature richly explores the meaning and usage of online SNS friendships, this work is some of the first to demonstrate that adolescents are willing to be online friends with a physician or researcher [8,26,29-31]. The worry that adolescents may not be willing to accept a FR from a researcher or health professional on an SNS such as Facebook or MySpace, may be less warranted than previously thought. The results of these two studies support the feasibility of friending adolescents on SNSs for research purposes. Nearly half of the adolescents in Study 1 who were unaware of their participation in a research study accepted a FR from an unknown physician's profile, three-quarters of whom maintained that online friendship nine months later. In Study 2, almost all of the adolescents 
were willing to accept a FR from a researcher and maintain contact for one year as part of their participation in a research study.

These results extend earlier work that first demonstrated young adults are willing to engage with medical professionals and researchers on SNSs. A prior study found that an unsolicited comment via SNS from a medical professional influenced display of risk behaviors online, thereby suggesting adolescents are receptive to interacting with medical professionals on SNSs [5]. Further, the majority of college students' networks are comprised of superficial relations, ie, acquaintances and activity-based friends [29]. From this current analysis, it appears that adolescents may in fact be willing to interact with clinicians and researchers online in a sustained manner over time.

These two studies are comparable for two reasons. First, the friending process on MySpace is almost the same as that on Facebook. Second, participants in both studies were 18 years old and therefore on par developmentally.

Despite these similarities, there are several differences between Study 1 and Study 2 that may account for the different FR acceptance and retention rates observed in these two studies. First, participants in Study 1 were unaware they were sent a FR as part of a research study. In Study 2, participants were told that they would receive a FR prior to receiving it on Facebook. Since the majority of college students' online networks are not composed of strangers, but rather acquaintances and activity-based friends, the preannouncement of the impending FR may have improved acceptance and retention rates by providing a sense of relationship, albeit impersonal and superficial, between researcher and adolescent.

Second, while a strength of this overall analysis, these studies were conducted using different SNSs and adolescent populations. Participants in Study 1 were general MySpace users, while participants in Study 2 were current college students with Facebook profiles. Further, Facebook is organized into networks whereas MySpace is not. The fact that the research profile used to send FRs in Study 2 was registered in the same network as the research participants may have engendered a sense of trust and familiarity resulting in higher FR acceptance rates. These differences may contribute to the varying acceptance rates between the two studies.

\section{Ethical considerations}

These new research methods may raise questions about the ethical use of social media websites for research purposes. First, concerns regarding confidentiality are important to consider. Many teens may post personal and identifying information in a public manner on their SNS profile, such as name, photographs, and contact information [32]. Despite this personal disclosure, it is important for researchers to maintain the same confidentiality practices when using SNS for research purposes as they do when engaging in other human subjects research [33]. Second, understanding the nuances of online SNS friendships is important. While some have argued that research participants may misconstrue the reception of a friend request on social media as an offer of a close or prolonged friendship, the literature suggests otherwise. For users of social media, "friending" implies a weak-tie relationship characteristic of acquaintances; many users are mere associates of their online friends [26]. Therefore, researchers should present an accurate and true identity on SNS, but need not be concerned that participants will misinterpret the intentions of the friend request [34].

\section{Limitations}

There are several limitations to this analysis. First, the authors acknowledge that these studies assessed the feasibility of friending on SNSs, rather than online communication via SNSs. SNSs offer many communication formats, including public postings, private messaging, and real time chatting. It remains unknown whether the numerous modes of communication via SNS may also be novel research tools. However, given today's current focus on online privacy, friending research participants is likely a critical step for establishing future dialogue with adolescents on SNSs.

Second, we provide data that may prompt comparisons of FR acceptance and retention rates between MySpace and Facebook. However, given the varying cultures of each SNS, the extent to which our findings from each study can be generalized and compared to other SNSs is not known. Third, we only examined the online behaviors of 18-year-olds and generalizing our results to younger adolescents or adult research participants may not be warranted.

\section{Conclusion}

Friending adolescents online is a useful and feasible research method for engaging participants online. These analyses illustrate that this can be done on different sites at different time points, providing benchmarks for friending acceptance and retention rates. Because the friending process allows profile content, including demographic and personal contact information to be mutually viewable, research teams may find this helpful for identifying and recruiting potential research participants. Researchers may also increase the transparency of their work and goals by posting relevant information on their own profile for participants to view.

For those interested in friending adolescents online for research purposes, we suggest notifying adolescents prior to sending a FR. Further, using a legitimate research profile registered in the same network as the research 
participant may also improve FR acceptance and retention rates. Doing so will also help to ensure ethical considerations regarding social media research. Finally, in both studies, we did not communicate publicly on participants' SNS pages. In doing so, we may have maintained credibility as professionals who were not trying to engage with participants at the friendship level by writing on their Wall or in casual communication. This approach may have supported our participants' willingness to maintain the online friendship longer term.

Future research should explore the feasibility of using the multiple communication modes built into SNSs for research purposes, such as scheduling appointments or delivering surveys. We are confident that SNSs like MySpace and Facebook are a feasible tool for health professionals and researchers looking to maintain an online connection with adolescents for research purposes.

\section{Competing interests}

The authors declare they have no competing interests.

\section{Authors' contributions}

LNB conceptualized the study, completed the analysis, and drafted and edited the manuscript. DAC participated in conceptualizing the study and editing the manuscript. MAM participated in conceptualizing the study and data analysis, and editing the manuscript. All authors read and approved the final manuscript.

\section{Acknowledgements}

The authors would like to thank Bradley Kerr, Katie G Egan, Michael J Swanson, and Lauren A Jelenchick for their assistance with data collection and manuscript preparation.

Received: 18 December 2013 Accepted: 16 April 2014

Published: 2 May 2014

\section{References}

1. Sun, $P$, Unger, JB, Palmer, PH, Gallaher, $P$, Chou, CP, Baezconde-Garbanati, L, Sussman, S, \& Johnson, CA. (2005). Internet accessibility and usage among urban adolescents in Southern California: implications for web-based health research. Cyberpsychology \& Behavior, 8(5), 441-53.

2. Lewis, K, Kaufman, J, \& Christakis, N. (2008). The taste for privacy: An analysis of college student privacy settings in an online social network. Journal of Comput-Mediated Communication, 14(1), 79-100.

3. Lenhart, A, Purcell, K, Smith, A, \& Zickuhr, K. (2010). Social Media \& Mobile Internet Use Among Teens and Young Adults. Washington, DC: Pew Internet \& American Life Project.

4. Moreno, MA, Vanderstoep, A, Parks, MR, Zimmerman, FJ, Kurth, A, \& Christakis, DA. (2009). Reducing at-risk adolescents' display of risk behavior on a social networking web site: a randomized controlled pilot intervention trial. Archives of Pediatrics and Adolescent Medicine, 163(1), 35-41.

5. Moreno, MA, Christakis, DA, Egan, KG, Brockman, LN, \& Becker, T. (2011). Associations Between Displayed Alcohol References on Facebook and Problem Drinking Among College Students. Archives of Pediatrics and Adolescent Medicine, 166(2), 157-63.

6. Ramo, DE, \& Prochaska, JJ. (2012). Broad reach and targeted recruitment using Facebook for an online survey of young adult substance use. J Med Internet Res, 14(1), e28.

7. Lenhart, A, Madden, M, \& Hitlin, P. Teens and Technology: Youth are Leading the Transition to a Fully Wired and Mobile Nation. In Pew Internet \& American Life Project. July 17 2005. Available from: http://www.pewinternet. org/Reports/2005/Teens-and-Technology.aspx.
8. Christofides, E, Muise, A, \& Desmarais, S. (2009). Information disclosure and control on Facebook: are they two sides of the same coin or two different processes? Cyberpsychology \& Behavior, 12(3), 341-345.

9. Hinduja, S, \& Patchin, JW. (2008). Personal information of adolescents on the Internet: A quantitative content analysis of MySpace. Journal of Adolescence, 31(1), 125-46.

10. Taraszow, T, Aristodemou, E, Shitta, G, Laouris, Y, \& Arsoy, A. (2010). Disclosure of personal and contact information by young people in social networking sites: An analysis using Facebook ${ }^{\mathrm{TM}}$ profiles as an example. International Journal of Media Cultural Politics, 6(1), 81-101.

11. Acquisti, A, \& Gross, R. (2006). Imagined communities: Awareness, information sharing, and privacy on the Facebook. Lecture Notes in Computer Science, 4258, 36-58.

12. Moreno MA, Parks MR, Zimmerman FJ, Brito TE, Christakis DA. (2009). Display of health risk behaviors on MySpace by adolescents: prevalence and associations. Archives of Pediatrics and Adolescent Medicine, 163(1), 27-34.

13. Moreno, MA, Brockman, LN, Wasserheit, JN, \& Christakis, DA. (2012). A pilot evaluation of older adolescents' sexual reference displays on Facebook. Journal of Sex Research, 39(3), 295-304.

14. Barnard, A. Facebook Agrees to More Safeguards, in New York Times. New York, NY: October 17, 2007. Accessed from: http://www.nytimes.com/2007/ 10/17/nyregion/17facebook.html.

15. Stone, B. States Fault MySpace on Predator Issues, in New York Times. New York, NY: May 15, 2007. Accessed from: http://www.nytimes.com/2007/05/ 15/technology/15myspace.html.

16. Nakamura, L, \& Chow-White, P. (2011). Race after the Internet. New York: Routledge.

17. Hargittai, E. (2007). Whose space? Differences among users and non-users of social network sites. Journal of Computer-Mediated Communication, 13(1), 276-297.

18. Watkins, SC. (2009). The young and the digital: what the migration to socialnetwork sites, games, and anytime, anywhere media means for our future (p. xxi-249). Boston, MA: Beacon Press.

19. Boyd, D. Viewing American class divisions through Facebook and MySpace, in Apophenia Blog. June 24, 2007. Accessed from: http://www.danah.org/ papers/essays/ClassDivisions.html.

20. Schulenberg, JE, \& Maggs, JL. (2002). A developmental perspective on alcohol use and heavy drinking during adolescence and the transition to young adulthood. Journal of Studies on Alcohol. Supplement, $14,54-70$.

21. Kunkel, D, Eyal, K, Finnerty, K, Biely, E, \& Donnerstein, E. (2005). Methodology, in Sex on TV:4 (pp. 14-16). Kaiser Family Foundation.

22. Kunkel, D, Eyal, K, \& Donnerstein, E. (2007). Sexual socialization messages on entertainment television: comparing content trends 1997-2002. Media Psychology, 9(3), 595-622.

23. Zou, G. (2004). A modified poisson regression approach to prospective studies with binary data. American Journal of Epidemiology, 159(7), 702-706.

24. Lumley, T, Kronmal, R, \& Ma, S. (2006). Relative Risk Regression in Medical Research: Models, Contrasts, Estimators, and Algorithms. In UW Biostatistics Working Paper Series. Working Paper \#293.

25. Ellison, NB, Steinfield, C, \& Lampe, C. (2007). The benefits of Facebook "friends": social capital and college students' use of online social network sites. Journal of Computer-Mediated Communication, 12(4), 1143-1168.

26. MySpace, LLC. (2012). Age Requirements. Available from: http://myspace.desk. com/customer/portal/articles/518684-age-requirements.

27. Buffardi, LE, \& Campbell, WK. (2008). Narcissism and social networking Web sites. Personality and Social Psychology Bulletin, 34(10), 1303-14.

28. Manago, AM, Taylor, T, \& Greenfield, PM. (2012). Me and my 400 friends: the anatomy of college students' Facebook networks, their communication patterns, and well-being. Developmental Psychology, 48(2), 369-80

29. Debatin, B, \& Lovejoy, JP. (2009). Facebook and online privacy: attitudes, behaviors, and unintended consequences. Journal of Computer-Mediated Communication, 15(1), 83-108.

30. Ross, C, Orr, ES, Sisic, M, Arseneault, JM, Simmering, MG, \& Orr, R. (2009). Personality and motivations associated with Facebook use. Computers in Human Behavior, 25(2), 578-586. 
31. Sledgianowski, D, \& Kulviwat, S. (2009). Using social network sites: The effects of playfulness, critical mass and trust in a hedonic context. Journal of Computer Information Systems, 49(4), 74-83.

32. Lenhart, A, \& Madden, M. (2007). Teens, Privacy and Online Social Networks. Washington, DC: Pew Internet and American Life Project.

33. Moreno, MA, Fost, NC, \& Christakis, DA. (2008). Research ethics in the MySpace era. Pediatrics, 121(1), 157-61.

34. Moreno, MA, Goniu, N, Moreno, PS, \& Diekema, D. (2013). Ethics of social media research: common concerns and practical considerations. Cyberpsychology \& Behavior, 16(9), 708-13.

doi:10.1186/2194-0827-2-1

Cite this article as: Brockman et al:: Friending adolescents on social networking websites: a feasible research tool. Journal of Interaction Science 2014 2:1.

\section{Submit your manuscript to a SpringerOpen ${ }^{\circ}$} journal and benefit from:

- Convenient online submission

- Rigorous peer review

- Immediate publication on acceptance

- Open access: articles freely available online

- High visibility within the field

- Retaining the copyright to your article 